ment, Texel).

As the humoral response after natural CDV infection persists for several years, and perhaps even for life, in the dogs, and assuming that this also holds true for pinnipeds we might conclude that CDV does not commonly infect pinniped species. Because acquired immunity to this virus is apparently not present in these animals, there is a potential risk that seals in the Canadian and American seas may also be subject to CDV infection. Further seroepizootiological studies among pinniped species at different locations, and biological and biochemical comparison of virus mals may elucidate the origin of the outbreak of CDV infection in seals.

A. D. M. E. Osterhaus

J. Groen

P. DE VRIES

F. G. C. M. UYTDEHAAG National Institute of Public Health and

Environmental Protection,

PO Box 1, 3720 BA Bilthoven,

The Netherlands

National Veterinary Institute,

B. KLINGEBORN

Uppsala, Sweden

Department of Fish and Game,

Fairbanks, Alaska, USA

1. Statement of the emergency working conference on seal deaths in the Baltic and North Seas, 11 August 1988, London. . Osterhaus, A.D.M.E. Nature 334, 301-302 (1988)

3. Osterhaus, A.D.M.E. \& Vedder, E.J. Nature 335, 20 (1988)

De Vries, P., UytdeHaag, F.G.C.M. \& Osterhaus, A.D. M. J gen Virol 69, 2071-2083 (1988)

5. Appel, M. J. gen. Virol. 41, 385-393 (1978)

\section{Confirmation of cause of recent seal deaths}

SIR-Osterhaus and Vedder recently presented serological evidence associating canine distemper virus (CDV) or a closely related morbillivirus with the current high mortality in European seals (Nature 335, $30 ; 1988)$. Here, we confirm such a virus as the cause of the deaths and report the isolation of a morbillivirus from an affected seal.

We have necropsied 17 dead or seriously ill seals (Phoca vitulina) found on the coast of Northern Ireland since 3 August 1988. Our main necropsy findings were severe pneumonia, encephalitis and ophthalmitis. Histopathological brain lesions were typical of a viral encephalitis isolates from terrestrial and aquatic mam-

and we saw many intracytoplasmic and intranuclear acidophilic inclusion bodies ( $a$ in the figure) characteristic of CDV infection in neurons and astrocytes (Dugworth, D.L. in Pathology of Domestic Animals (eds Jubb, J. et al.) Academic, London; 1985). Pulmonary lesions were characterized by diffuse interstitial pneumonia. There was proliferation of type II pneumonocytes resulting in syncytia formation in the lungs of several seals. Although such changes are consistent with CDV infection, we did not see inclusion bodies in the lungs of any of the seals.

We examined frozen sections of pneumonic lung from six seals $(P$. vitulina) by indirect immunofluorescence using a polyclonal hyperimmune antiserum raised in rabbits to the Onderstepoort strain of CDV. Coverslip preparations of cell cultures infected with this strain were used as positive controls. We detected positive immunofluorescence in the lungs of three of the six seals. Immunofluorescence had mainly a diffuse cytoplasmic distribution pattern but large intracytoplasmic inclusion bodies also stained.

We also isolated a virus by culture of cells from the kidney of a seal that had encephalitis and ophthalmitis. The virus produces cell vacuolation and syncytia, characteristic effects of a morbillivirus. Positive immunofluorescence using our hyperimmune antiserum confirms the cultured virus as a morbillivirus ( $b$ in the figure).

Our finding of histopathological lesions and inclusion bodies similar to those of CDV infection in other species, and demonstration of morbillivirus antigen in diseased lung and isolation of a morbillivirus from an affected seal provide very strong evidence for an aetiological role of CDV or a closely related morbillivirus as a primary cause of seal mortality. Further characterization of the virus is in progress.

S. KENNEDY

J. A. SMYTH

S. J. MCCullough

G. M. Allan

F. MCNeIlly

Veterinary Research Laboratories,

Stormont,

Belfast BT43SD, Northern Ireland

Department of Pathology,

Queen's University,

Belfast BT7 INN, Northern Ireland

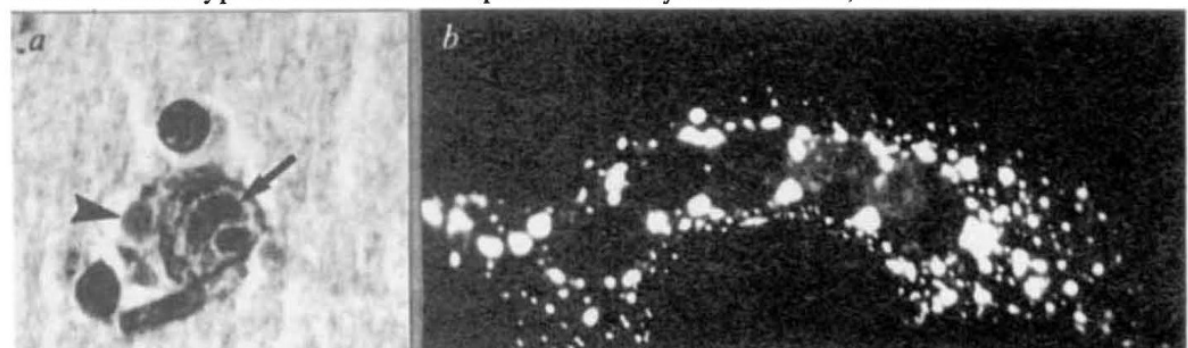

$a$, Intranuclear (arrow) and intracytoplasmic (arrowhead) inclusion bodies in neuron of seal with encephalitis. $b$, Morbillivirus antigen in syncytium in seal kidney cell culture.

\section{Ancient carbon sources of atmospheric methane}

SIR-Sackett and Barber ${ }^{1}$ comment on the observation $^{2}$ that 32 per cent of atmospheric methane is ${ }^{14} \mathrm{C}$-free and therefore of an ancient or primordial origin. They quote quantitative aspects of the possible emission of unburnt methane to the atmosphere by the petroleum industry, and of the possible release of methane from fresh tar. They do not, however, give the quantities with which this would have to be compared to account for the observation.

The total mass of carbon in the form of atmospheric methane (at a volume proportion of 1.6 parts per million) is $3.37 \times$ $10^{15} \mathrm{~g}$, of which the 32 per cent that is not from locally recycled plant material accounts for $1.08 \times 10^{15} \mathrm{~g}$. Because atmospheric methane has an average lifetime (against oxidation) of 5-7 yr, 1.54-2.16x $10^{14} \mathrm{~g}$ of this quantity will be replaced each year. This compares with the "several" units of $10^{12} \mathrm{~g}$ suggested by Sackett and Barber to come from venting of unburnt methane by the petroleum industry, and five such units from the exposure of fresh tar. The petroleum industry thus seems to be responsible for at most a few per cent of the atmospheric methane.

Addition of methane to the atmosphere has a far larger greenhouse effect ${ }^{3}$ than a similar quantity of carbon in the form of $\mathrm{CO}_{2}$, as the $\mathrm{CO}_{2}$ infrared bands are already practically saturated. The natural seepage of methane from gas fields provides a large contribution, which is reduced, demonstrably in some cases, by the production of gas from those fields. Because the gas produced in this way is generally burnt to $\mathrm{CO}_{2}$ (and water) it will contribute much less to the greenhouse effect than if it were allowed to seep into the atmosphere as methane. Therefore, it is possible that the gas-producing industry (although not the oil industry) may in fact be helping to restrain the greenhouse effect.

It may also be interesting to relate the quantity of ${ }^{14} \mathrm{C}$-free carbon now observed to the supply of juvenile carbon that appears to have entered the atmosphere over geological times and resulted in the deposition of carbon in the sediments, in carbonates and other forms. The total amount of carbon so laid down in the past 500 million years is generally judged to be of the order of $10^{23} \mathrm{~g}$. An annual supply rate of $2.16 \times 10^{14} \mathrm{~g}$ (derived above) would provide $1.3 \times 10^{23} \mathrm{~g}$ in this time.

Institute of Astronomy,

T. GOLD

Madingley Road,

Cambridge CB3 OHA,

$U K$

1. Sackett, W.M. \& Barber, T.R. Nature 334, 201 (1988)

Lowe, D.C. et al. Nature 332, 522-525 (1988).

3. Cicerone, R.J. Nature 334, 198 (1988). 